\title{
Técnica de inseminação artificial por tração cervical e função luteal em ovelhas Santa Inês após estro sincronizado com cloprostenol ou progestágeno-eCG
}

Technique of artificial ins emination by cervical traction and luteal function in Santa Inês ewes after synchronized estrus with cloprostenol or progestagen-eCG

\section{Caio Tácito Gomes Alvares ${ }^{1}$, Carla Cristina Romano ${ }^{2}$, Felipe Zandonadi Brandão ${ }^{3}$, Jurandir Ferreira da Cruz}

\author{
${ }^{1}$ Universidade Estadual de Santa Cruz, Departamento de Ciências Agrárias e Ambientais, Ilhéus, Bahia, Brasil \\ Rodovia Jorge Amado, km 16, Salobrinho, Ilhéus, BA, 7336805696 \\ ${ }^{2}$ Universidade Estadual de Santa Cruz, Departamento de Ciências Biológicas, Ilhéus, Bahia, Brasil \\ ${ }^{3}$ Universidade Federal Fluminense, Niterói, Rio de Janeiro, Brasil \\ ${ }^{4}$ Universidade Estadual do Sudoeste da Bahia, Vitória da Conquista, Bahia, Brasil
}

\begin{abstract}
Resumo
Avaliou-se a influência do protocolo de sincronização de estro na execução da técnica de inseminação artificial por tração cervical (ITC) e subsequente perfil sérico de progesterona em ovelhas Santa Inês. Um grupo foi submetido a duas aplicações de cloprostenol com sete dias de intervalo (PGF2 $\alpha$, $n=43$ ), outro grupo foi sincronizado com MAP-eCG (P4-eCG, $n=40)$ e ovelhas ao estro natural (EN, $\mathrm{n}=10$ ) formaram grupo controle. Com as ovelhas contidas em estação, foi realizada a técnica ITC utilizando um aplicador metálico e mandril. Foram avaliadas a profundidade de penetração e intensidade de reação da ovelha ao manejo da técnica. Dosagens de estradiol e progesterona foram feitas antes e depois da ITC/estro natural, respectivamente. Os níveis de estradiol foram superiores com P4-eCG vs PGF2 $\alpha(\mathrm{P}<0,05)$, mas não houve influência do protocolo na execução da técnica. Ocorreram $83,5 \%$ de deposição intrauterina do sêmen, o tempo médio foi de 32 segundos e 90,7\% das ovelhas apresentaram reação fraca ou moderada durante o manejo. Os níveis de progesterona foram semelhantes entre ITC e estro natural. Independente do protocolo hormonal, a técnica ITC em ovelhas Santa Inês mantidas em estação mostrou-se viável no tempo de penetração da cérvice e deposição intrauterina do sêmen, não comprometendo a função luteal subsequente.
\end{abstract}

Palavras-chave: aplicador expansor, cérvice, fisiologia reprodutiva, Synchrovine ${ }^{\circledR}$

\begin{abstract}
The influence of estrus synchronization protocol was evaluated on the artificial insemination technique by cervical traction (ICT) and subsequent progesterone serum profile in Santa Inês ewes. One group was submitted to two applications of cloprostenol seven days apart (PGF2 $\alpha, n=43)$, another group was synchronized with MAP-eCG (P4-eCG, $n=40)$ and ewes at natural estrus (NE, $n=10)$ formed a control group. With the ewe contained in the stand position, the ICT technique was performed using a metal applicator and mandrel. Depth of penetration and reaction intensity of the ewe to the handling of the technique were evaluated. Dosages of estradiol and progesterone were performed before and after ICT / natural estrus, respectively. Estradiol levels were higher with P4-eCG vs PGF2 $\alpha(P<0.05)$, but there was no influence of the protocol on the execution of the technique. There was $83.5 \%$ of intrauterine semen deposition, the average time was 32 seconds and $90.7 \%$ of the ewes showed a weak or moderate reaction during handling. Progesterone levels were similar between ICT and natural estrus. Regardless of the hormonal protocol, the ICT technique in Santa Inês ewes kept in standing position proved to be viable in the time of cervix penetration and intrauterine sêmen deposition, without compromising the subsequent luteal function.
\end{abstract}

Keywords: Expanding catheter, cervix, reproductive physiology, Synchrovine ${ }^{\circledR}$

\section{Introdução}

O avanço genético de um rebanho ovino pode ser alcançado utilizando-se biotécnicas de reprodução animal, como a inseminação artificial (IA). Esta técnica permite que reprodutores de elevado mérito 
Alvares et al. Técnica de inseminação artificial por tração cervical e função luteal em ovelhas Santa Inês após estro sincronizado com cloprostenol ou progestágeno-eCG

genético possam servir a um número superior de fêmeas por estação reprodutiva, quando comparada com a monta natural, otimizando o uso desses animais. Aliado a isso, o uso de protocolos de sincronização de estro permite, em nível comercial, reduzir a estação reprodutiva, controlar nascimentos, facilitar cuidados com a cria e desmame, padronizando lotes (Miranda et al., 2018; Rosasco et al., 2019). Protocolos hormonais a base de progestágenos e gonadotrofina coriônica equina (eCG) são amplamente utilizados em estudos de IA; o eCG contribui para elevação dos níveis séricos de estradiol, hormônio envolvido na dilatação da cérvice ao estro (Dias et al., 2001; Falchi e Scaramuzzi, 2013). Por outro lado, o uso de prostaglandina F2 $\alpha$ e seus análogos sintéticos, apesar de proporcionar índices reprodutivos inferiores, facilita o manejo e diminui custos (Hassani et al., 2018).

A técnica de inseminação artificial por via transcervical visa alcançar o útero sem traumas na cérvice em uma quantidade mínima de tempo, que viabilize o manejo a campo. Contudo, apesar de diversos estudos, a metodologia desta técnica após estro sincronizado não apresenta taxas de fertilidade aceitáveis em condições de campo (Alvarez et al., 2019). A anatomia cervical das ovelhas consiste em um entrave para o alcance do sucesso desta biotécnica, uma vez que a disposição irregular dos anéis dificulta a passagem dos aplicadores convencionais até o corpo do útero (Kershaw et al, 2005). Com isso, Halbert et al. (1990) idealizaram o sistema Guelph, que consiste na tração e fixação da cérvice para possibilitar a transposição de seus anéis, estando a ovelha em decúbito dorsal. Existem vários modelos de pipeta testados para inseminação transcervical. Moura et al. (2011) avaliaram em peças anatômicas um modelo aplicador metálico com um mandril de ponta romba que permite transpassar os anéis cervicais; uma vez retirado o mandril, a dose de sêmen é introduzida para inseminação. Modelo semelhante foi utilizado por Casali et al. (2017) para IA transcervical em ovelhas Corriedale mantidas com o quarto traseiro em um cavalete.

Apesar da potencialidade dessa técnica, alguns autores sugerem que a manipulação e tração da cérvice no momento da IA podem elevar a produção de PGF2 $\alpha$, de tal modo que ocorra invasão de neutrófilos e citocinas pró-inflamatórias no tecido luteal, provocando alterações no ambiente uterino, com consequente luteólise e morte embrionária (Wulster-Radcliffe e Lewis, 2002; Wulster-Radcliffe et al., 2004; Weems et al., 2006). Os resultados inconsistentes de fertilidade com a IA transcervical utilizando diferentes instrumentos sugere que sejam estudadas possíveis influências da técnica de IA no ambiente uterino. Na literatura são abordados estudos que associam a manipulação cervical à perda de fertilidade em ovelhas (Anel et al., 2006). Da mesma forma, torna-se necessária a viabilização de um manejo de IA que contribua para o bem-estar do animal e redução do tempo de execução, minimizando seu estresse (Martin e Ferasyi, 2016). Diante do exposto, este estudo teve como objetivo avaliar a execução da técnica de inseminação artificial por tração cervical (ITC) em ovelhas Santa Inês manejadas em estação após sincronização de estro com cloprostenol (PGF2 $\alpha$ ) ou progestágeno/eCG e a função luteal subsequente.

\section{Material e Métodos}

\section{Local, animais e tratamentos}

Todos os procedimentos experimentais utilizados neste estudo foram aprovados pela Comissão de Ética no Uso de Animais da Universidade Estadual do Sudoeste da Bahia (protocolo nº 68/2014). O estudo foi realizado na Estação Experimental Fazenda Almada, Núcleo de Ovinocultura Tropical,

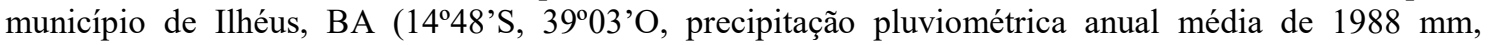
temperatura $23,4 \pm 1,5^{\circ} \mathrm{C}$ e umidade relativa $85,4 \pm 4,0 \%$ ). Foram utilizadas 93 ovelhas da raça Santa

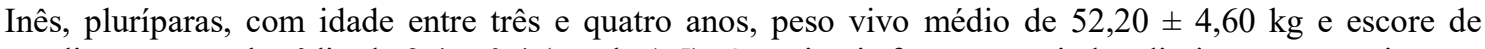
condição corporal médio de 3,4 $\pm 0,4$ (escala 1-5). Os animais foram manejados diariamente em piquetes de Brachiaria humidicola das $8: 00 \mathrm{~h}$ às 16:00h e suplementados com capim elefante (Pennisetum purpureum) e concentrado à base de milho e soja (15\% PB) na quantidade média de $400 \mathrm{~g} / \mathrm{cab} / \mathrm{dia}$; sal mineral (Guabiphos ovinos AE®, Guabi, Brasil) e água foram ad libitum. Do total de ovelhas, 83 foram separadas em dois grupos e submetidas a dois protocolos de sincronização do estro; no primeiro grupo (PGF2 $\alpha, n=43$ ) foram realizadas duas aplicações IM de $125 \mu \mathrm{g}$ de cloprostenol (Ciosin ${ }^{\circledR}$, MSD Saúde Animal, Brasil) com intervalo de sete dias; no segundo grupo ( $\mathrm{P} 4-\mathrm{eCG}, \mathrm{n}=40)$ ) foram inseridas esponjas intravaginais contendo 60mg de acetato de medroxiprogesterona (Progespon ${ }^{\circ}$, Zoetis, Brasil), mantidas

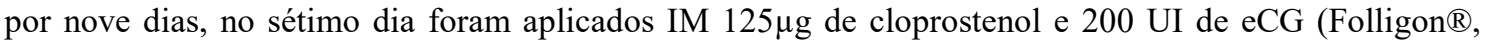
MSD Saúde Animal, Brasil). As ovelhas não submetidas aos protocolos hormonais foram utilizadas como grupo controle, ou seja, ao estro natural $(\mathrm{EN}, \mathrm{n}=10)$. A observação do estro ocorreu diariamente, duas 
Alvares et al. Técnica de inseminação artificial por tração cervical e função luteal em ovelhas Santa Inês após estro sincronizado com cloprostenol ou progestágeno-eCG

vezes ao dia, às 7:00h e 19:00h, com auxílio de rufiões vasectomizados.

\section{Técnica de inseminação artificial por tração cervical (ITC)}

As ovelhas foram submetidas à técnica de ITC 48-54 horas após segunda injeção de cloprostenol no protocolo PGF2 $\alpha$ (Olivera-Muzante et al., 2011) e 54-56 horas após retirada da esponja intravaginal no protocolo P4-eCG. Para manutenção das ovelhas em estação foi utilizado um brete com uma tábua de apoio ventral e os membros posteriores imobilizados com uso de cordas. Para realização da técnica ITC, após higienização da região vulvar, utilizou-se um espéculo vaginal de $15 \mathrm{~cm}$ e fonte de luz para visualização do óstio cervical; realizou-se a tração da cérvice até a abertura vulvar e fixação por meio de duas pinças Allis de $25 \mathrm{~cm}$ de comprimento; posteriormente foi utilizado um aplicador metálico de $12 \mathrm{~cm}$ contendo um mandril (Aplicador expansor ovino®, Alta Genetics, Brasil) para transpor os anéis cervicais, tanto quanto possível (Fig. 1); em seguida foi introduzida uma palheta de 0,25 mL contendo sêmen descongelado a $35-37^{\circ} \mathrm{C}$ por 30 segundos sem capacidade fecundante, por ter sido previamente submetido ao intenso choque térmico (descongelamento das doses a $37^{\circ} \mathrm{C} \mathrm{com} \mathrm{posterior} \mathrm{devolução} \mathrm{ao}$ botijão com nitrogênio líquido; uma gota de cada dose utilizada foi avaliada antes da utilização). Esta medida foi tomada para se permitir a execução da técnica ITC o mais próximo possível da realidade, tanto do manejo quanto dos componentes presentes na dose inseminante, sem a possível influência de conceptos no ambiente uterino (Shäfer-Somi, 2003). Dependendo da profundidade alcançada pelo aplicador, a deposição do sêmen foi caracterizada como: (1) cervical superficial (sêmen depositado na cérvice, até dois anéis), (2) cervical profunda (sêmen depositado na cérvice, acima de dois anéis, porém não alcançando o útero) ou (3) intrauterina. O tempo gasto para penetração da cérvice até o local de deposição do sêmen foi cronometrado; a intensidade de reação da ovelha ao manejo da contenção e execução da técnica foi classificada como: fraca (pouco a nenhum movimento ou balido a partir da contenção, mínima reação ao uso do espéculo, pinças ou aplicador), moderada (tentativas esparsas de se soltar da contenção, balidos isolados e reações esporádicas ao uso do espéculo, pinças ou aplicador) e forte (tentativas constantes de se soltar da contenção, balidos frequentes e reação imediata ao uso do espéculo, pinças ou aplicador).
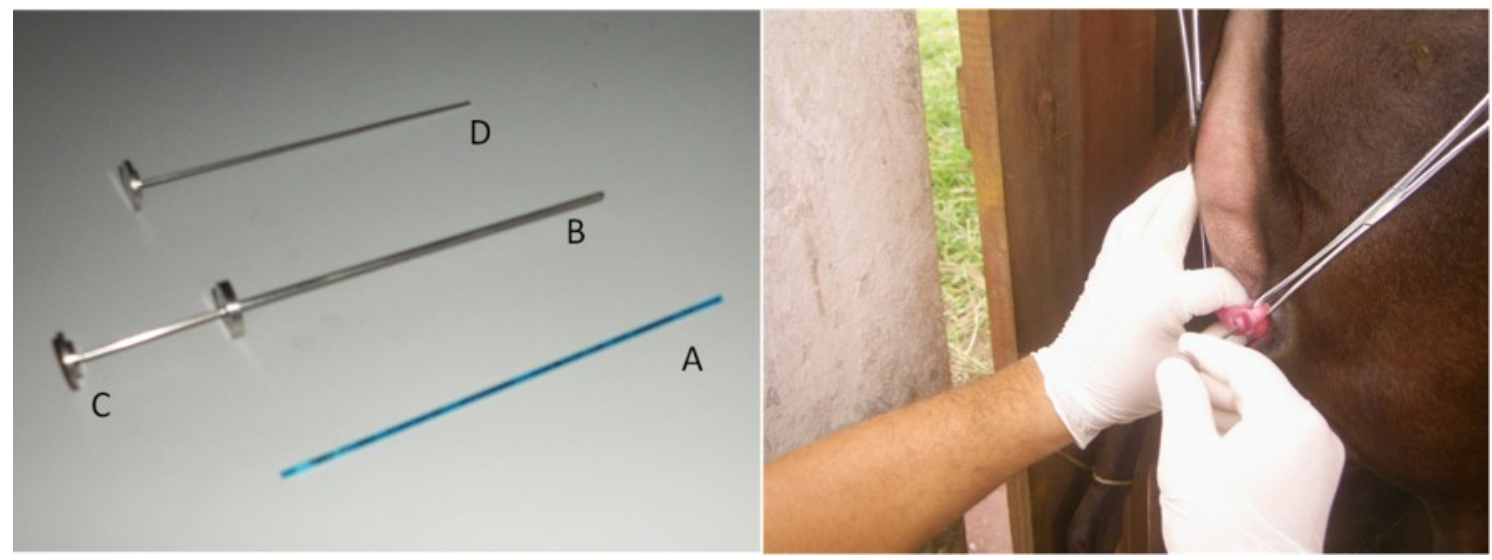

Figura 1. À esquerda: palheta 0,25mL (A), aplicador de sêmen (B), mandril para transposição cervical (C) e êmbolo para dep osição da dose inseminante (D); à direita, técnica de inseminação artificial por tração cervical (ITC), evidenciando fixação da cérvice junto a abertura vulvar com duas pinças Allis e penetração cervical com o aplicador e mandril.

\section{Dosagem hormonal}

Para determinação dos níveis séricos hormonais foram coletadas amostras de sangue de 10 ovelhas de cada grupo (PGF2 $\alpha$, P4-eCG e EN) nos dias -1 e 0 (17 $\beta$ estradiol) e nos dias 0, 3, 5, 12 e 17 (progesterona), sendo o “dia 0" referente à realização da técnica ITC ou, no caso do grupo EN, à ocorrência do estro. As amostras foram centrifugadas a $3000 \mathrm{rpm}$ por 10 minutos e o soro obtido foi armazenado em freezer a $-20^{\circ} \mathrm{C}$. A dosagem hormonal foi realizada com as amostras em duplicata por 
Alvares et al. Técnica de inseminação artificial por tração cervical e função luteal em ovelhas Santa Inês após estro sincronizado com cloprostenol ou progestágeno-eCG

Radioimunoensaio no Laboratório de Reprodução Animal da Universidade Federal Fluminense, RJ. Para quantificar o $17 \beta$ estradiol foi utilizado kit RIA DSL4800 (Beckman Coulter ${ }^{\circledR}$, Immunotech, República Tcheca), com sensibilidade de $2,2 \mathrm{pg} / \mathrm{mL}$ e coeficientes de variação intra-ensaio e interensaio de $8,9 \%$ e $12,2 \%$, respectivamente. Para dosagem da progesterona, o kit RIA IM1188 (Beckman Coulter ${ }^{\circledR}$, Immunotech, República Tcheca), com sensibilidade de $0,05 \mathrm{ng} / \mathrm{mL}$ e coeficientes de variação intra-ensaio e interensaio de $6,5 \%$ e $7,2 \%$, respectivamente.

\section{Análise estatística}

Os dados referentes ao local de deposição do sêmen e intensidade de reação da ovelha à ITC foram comparados pelo teste Qui-quadrado. As médias $( \pm \mathrm{dp})$ do tempo de penetração cervical foram comparadas pelo teste $\mathrm{F}$, enquanto que, para a comparação das dosagens hormonais foi utilizado o teste Student-Newman-Keuls (PROC GLM, SAS versão 9.1). As diferenças foram consideradas significativas com $\mathrm{P}<0,05$.

\section{Resultados}

\section{Dosagem de estradiol}

Os níveis séricos de $17 \beta$ estradiol nos dias -1 e 0 foram significativamente superiores em ovelhas submetidas ao protocolo P4-eCG, comparando-se com o PGF2 $\alpha(20,1 v s$ 9,1 pg/mL e 7,1 vs 2,9 pg/mL, respectivamente, $\mathrm{P}<0,05)$, porém, em ambos os protocolos foi observado um declínio acentuado do estrógeno entre os dias avaliados, o que não ocorreu com ovelhas ao estro natural, cujos níveis séricos foram similares (11,3 e 9,7 pg/mL) (Fig. 2).

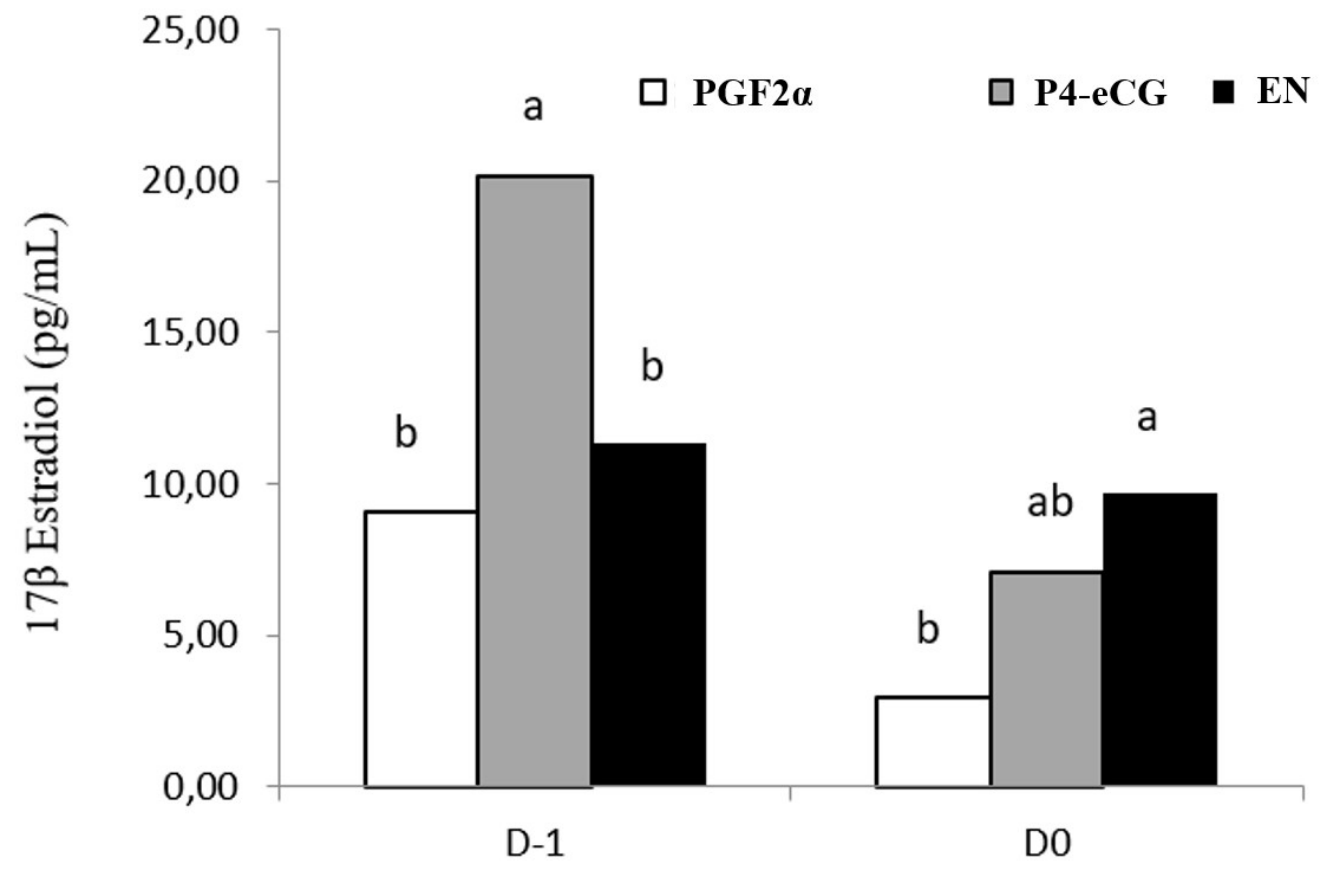

Figura 2. Níveis séricos de $17 \beta$ estradiol em ovelhas Santa Inês com estro sincronizado por cloprostenol $(\mathrm{PGF} 2 \alpha)$ ou progestágeno-eCG (P4-eCG) e submetidas à técnica de inseminação artificial por tração cervical (ITC) no dia zero (D0). Grupo controle com ovelhas ao estro natural (D0) e sem técnica de ITC (EN). $\mathrm{P}<0,05$ pelo teste $\mathrm{SNK}$. 
Alvares et al. Técnica de inseminação artificial por tração cervical e função luteal em ovelhas Santa Inês após estro sincronizado com cloprostenol ou progestágeno-eCG

\section{Execução da técnica de ITC e dosagem de progesterona}

Não houve influência dos protocolos de sincronização do estro em nenhum dos parâmetros avaliados para a técnica de ITC (Tab. 1). Do total de ovelhas inseminadas, em apenas 16,5\% ocorreu deposição cervical superficial, não sendo possível ultrapassar o segundo anel, contudo, uma vez superada esta barreira, a deposição intrauterina do sêmen foi viabilizada em 83,5\% das ovelhas; o tempo médio de manipulação da cérvice foi de 32 segundos, com variação de oito a 90 segundos (tempos mínimo e máximo, respectivamente).

Considerando todas as ovelhas submetidas à técnica, a ocorrência de reações fracas e moderadas à manipulação cervical foi de $41,9 \%$ e $48,8 \%$, respectivamente, com apenas $9,3 \%$ das fêmeas apresentando reações fortes (Tab. 1).

Tabela 1. Local de deposição do sêmen, tempo médio de penetração cervical e intensidade da reação ao manejo da técnica de inseminação artificial por tração cervical (ITC) em ovelhas Santa Inês com estro sincronizado por cloprostenol (PGF2 $\alpha$ ) ou progestágeno-eCG (P4-eCG)

\begin{tabular}{ccc}
\hline Grupo & PGF2 $\alpha$ & P4-eCG \\
\hline N & 43 & 40 \\
Local de deposição do sêmen (\%) & 13,0 & 20,0 \\
Cervical superficial (até dois anéis) & 0,0 & 0,0 \\
Cervical profunda (após dois anéis) & 87,0 & 80,0 \\
Intrauterina & $32,68 \pm 25,28$ & $30,94 \pm 14,68$ \\
Tempo médio de penetração cervical (s) & & \\
Intensidade da reação da ovelha ao manejo & 39,1 & 45,0 \\
da técnica de ITC (\%) & 52,2 & 45,0 \\
Fraca & 8,7 & 10,0 \\
Moderada & &
\end{tabular}

$\mathrm{P}>0,05$ pelo Teste $\mathrm{F}$.

Os níveis séricos de progesterona das ovelhas com estro sincronizado e submetidas à técnica de ITC (PGF2 $\alpha$ ou P4-eCG) foram equivalentes às condições fisiológicas observadas em ovelhas com estro natural e sem manipulação cervical $(\mathrm{EN})$, não divergindo entre si ao longo do ciclo estral subsequente (P>0,05, Fig. 3).

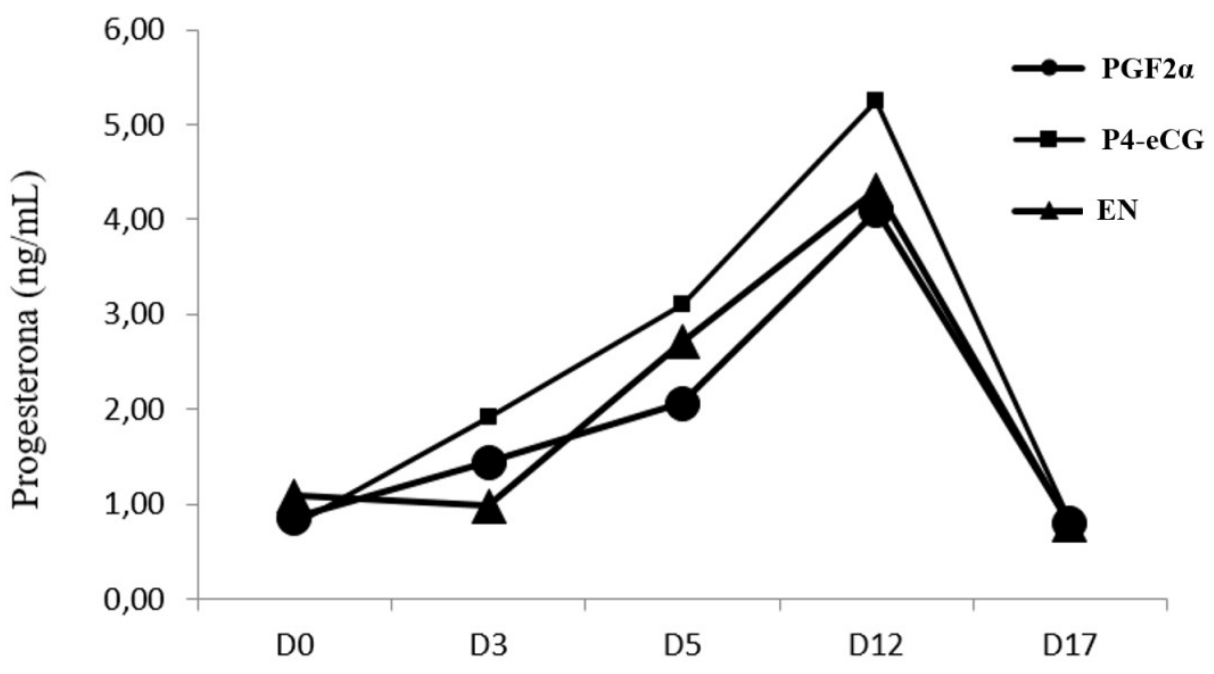

Figura 3. Níveis séricos de progesterona em ovelhas Santa Inês com estro sincronizado por cloprostenol $(\mathrm{PGF} 2 \alpha)$ ou progestágeno-eCG (P4-eCG) e submetidas à técnica de inseminação artificial por tração cervical (ITC) no dia zero (D0). Grupo controle composto por ovelhas ao estro natural (D0) e sem a técnica ITC $(\mathrm{EN})$. ( $\mathrm{P}>0,05$ pelo teste $\mathrm{SNK})$. 
Alvares et al. Técnica de inseminação artificial por tração cervical e função luteal em ovelhas Santa Inês após estro sincronizado com cloprostenol ou progestágeno-eCG

\section{Discussão}

Os níveis séricos de estradiol mais elevados no grupo P4-eCG já eram esperados. O eCG, por sua ação estimulante sobre o crescimento folicular, contribui para a elevação dos níveis de estrógeno (Dias et al., 2001). Contudo, a expectativa de maior penetrabilidade do aplicador na cérvice das ovelhas do grupo P4-eCG em função do estradiol não se concretizou, possivelmente a redução acentuada desses níveis no D0 em ambos os protocolos tenha eliminado essa influência. Embora o grau de relaxamento da cérvice durante o estro esteja associado ao aumento do estradiol, também se faz necessária a participação dos hormônios gonadotróficos (FSH e LH) e oxitocina na geração de uma cascata bioquímica, que resulta na produção de fatores parácrinos, como a prostaglandina E2 (PGE2), atuante no rearranjo das fibras celulares da musculatura lisa cervical (Falchi e Scaramuzzi, 2013), variáveis não mensuradas neste estudo. Da mesma forma, considerando que a dilatação cervical ao estro também esteja relacionada à degradação do colágeno na matriz extracelular, que envolve sinalização da interleucina 8 (IL-8) para infiltração de neutrófilos (Croy et al., 1999), a penetrabilidade cervical similar entre os grupos apresentada neste estudo está de acordo com relato de Mitchell et al. (2002), que revelou níveis de IL-8 semelhantes no tecido cervical de ovelhas com estro sincronizado por PGF $2 \alpha$ ou progestágeno.

A escolha de um protocolo hormonal vai além de seu desempenho, envolve também aspectos como economicidade, praticidade, bem estar e ética, visando maior adesão de produtores a esta biotécnica reprodutiva. Nesse contexto, os estudos voltados para o uso de PGF2 $\alpha$, mesmo se alcançando resultados reprodutivos inferiores ao $\mathrm{P} 4-\mathrm{eCG}$, são motivados por essas questões: rápida metabolização, sem uso de antibióticos ou ocorrência de vaginite, sem produção de anticorpos, a exemplo do que ocorre com o eCG (Hassani et al., 2018).

Mesmo com a vasta literatura apresentando diversos métodos para execução da técnica de IA transcervical, combinando protocolos hormonais, contenções, instrumentos e até mesmo fármacos dilatadores, os resultados de fertilidade são inconsistentes (Alvarez et al., 2019; Purdy et al., 2020). Portanto, estudar a eficiência da técnica de IA em si e suas possíveis consequências na ovelha, antes mesmo de se mensurar os índices reprodutivos, torna-se de grande utilidade. Como exemplo, Manes et al. (2014) relataram que a presença da esponja intravaginal na ovelha, por si só, sem progestágeno, já provoca diminuição da fertilidade. Campbell et al. (1996) identificaram diversas lesões teciduais na cérvice de ovelhas submetidas a eutanásia após técnica de inseminação cervical.

Neste estudo, independente do protocolo hormonal utilizado, a penetrabilidade cervical com o aplicador expansor foi elevada: $83,5 \%$ de deposição intrauterina do sêmen nas ovelhas submetidas à técnica ITC. Este resultado difere do alcançado por Casali et al. (2017) que relataram 28,8\% de deposição do sêmen além de $4 \mathrm{~cm}$ da cérvice e apenas 7,7\% intrauterina, utilizando modelo semelhante de aplicador e pinças para tração cervical. A irregularidade cervical em ovelhas é maior no segundo e terceiro anéis, o que compromete a profundidade de penetração de alguns modelos de aplicadores (Halbert et al., 1990). O uso de um modelo convencional de aplicador proporcionou a penetração intrauterina em menos de 7\% das cérvices, determinando a profundidade média de penetração em torno de $36,0 \mathrm{~mm}$, o que é condizente com a distância entre o segundo anel e o óstio cervical (Kershaw et al., 2005). O uso do sistema Guelph de ITC possibilitou maior penetrabilidade em comparação aos aplicadores convencionais, alcançando $40,4 \%$ e 44,7\% de deposição cervical profunda e intrauterina, respectivamente (Naqvi et al., 1998).

O tempo médio de penetração cervical também se mostrou eficiente com ambos os protocolos hormonais, em média 32 segundos. Os tempos de execução da IA utilizando o método de tração cervical são variados na literatura, entre 3 e 8 minutos (Naqvi et al., 1998; Casali et al., 2017), mas essa variação pode ser atribuída a diversos fatores, como raça, idade, período pós-parto e habilidade do inseminador (Kershaw-Young et al., 2010; Richardson et al., 2012). A forma de contenção do animal também é um fator que pode interferir nos resultados da técnica de ITC. Apesar do relato de que conter a ovelha em decúbito dorsal facilita a penetração do cateter através do canal cervical (Halbert et al., 1990a), no presente estudo, a contenção da ovelha em estação coincidiu com elevada penetrabilidade da cérvice. Dessa forma, acredita-se que o manejo de contenção da ovelha neste estudo possa ter contribuído para este reduzido tempo de penetração cervical. A intensidade de reação das ovelhas ao manejo e técnica de ITC foi considerada aceitável para condições de bem estar do animal. Casali et al. (2017) utilizaram contenção com cavalete e quarto traseiro da ovelha suspenso, e identificaram tempo de execução da ITC bem superior à laparoscopia, o que inviabiliza sua aplicação em programas de IATF em larga escala. Entretanto, os custos da laparoscopia, bem como o estresse gerado (jejum prévio, sedação, insuflação, posição céfalo-declive), limitam sua utilização (Alvarez et al., 2019). 
Alvares et al. Técnica de inseminação artificial por tração cervical e função luteal em ovelhas Santa Inês após estro sincronizado com cloprostenol ou progestágeno-eCG

Ovelhas que apresentem possíveis anormalidades no padrão secretório de progesterona podem ter a fertilidade comprometida (Barnes et al., 2000). Assim, embora exista relato de que o protocolo a base de PGF2 $\alpha$ possa interferir no ciclo estral por comprometer a formação do corpo lúteo (CL) e, consequentemente, a produção de progesterona (Wiley et al., 1997), no presente estudo este fato não foi verificado, uma vez que os níveis de progesterona ficaram dentro da normalidade, comparados com ovelhas ao estro natural, corroborando estudo prévio realizado com o mesmo protocolo (Fierro et al., 2011).

$\mathrm{O}$ fato de se ter utilizado sêmen sem capacidade fecundante, além da tentativa de se manter os componentes do sêmen o mais próximo possível da realidade, permitiu a avaliação da influência da técnica ITC no funcionamento luteal subsequente, sem a interferência de possíveis embriões que, por secretarem quantidades expressivas de citocinas, enzimas, prostaglandinas e hormônios para reconhecimento materno (Schäfer-Somi, 2003), certamente proporcionariam alterações no ambiente uterino, descaracterizando, portanto, este ciclo estral. Com isso, foi possível observar que, independente do protocolo hormonal, a técnica ITC utilizada neste estudo, por si, não alterou a fisiologia do ciclo estral subsequente quanto aos aspectos luteotróficos. Existem relatos de que a manipulação e tração da cérvice no momento da IA podem elevar a produção de PGF2, de tal modo que ocorra invasão de neutrófilos e citocinas pró-inflamatórias (Th1) no tecido luteal, provocando alterações no ambiente uterino, com consequente luteólise (Wulster-Radcliffe e Lewis, 2002; Wulster-Radcliffe et al., 2004; Weems et al., 2006). Ainda, a possibilidade de estresse, tanto pelo manejo quanto pela manipulação cervical, podem influenciar o eixo hipotalâmico-hipofisário-adrenal e inibir GnRH, LH ou secreção de progesterona (Emsem et al., 2011; Dobson et al., 2012). Entretanto, esta manipulação parece não ter comprometido a formação e manutenção do corpo lúteo, uma vez que a secreção de progesterona ao longo do ciclo estral foi mantida dentro dos parâmetros fisiológicos, demonstrados pelas ovelhas ao estro natural.

\section{Conclusões}

A execução da técnica de inseminação artificial por tração cervical (ITC) em ovelhas Santa Inês, mantidas em estação, após sincronização de estro com cloprostenol ou progestágeno-eCG, mostrou-se viável: proporcionou reações predominantemente leves/moderadas ao manejo, elevada taxa de deposição intrauterina do sêmen e reduzido tempo médio de penetração cervical, independente da diferença de níveis de estradiol apresentada entre os protocolos hormonais.

Os níveis séricos de progesterona similares aos apresentados por ovelhas ao estro natural sugerem que o manejo da técnica de ITC não comprometeu a função luteal subsequente.

\section{Agradecimentos}

À FAPESB, pelo apoio financeiro.

\section{Referências}

Alvarez M, Anel-Lopez L, Boixo JC, Chamorro C, Neila-Montero M, Montes-Garrido R, Paz P Anel L. Current challenges in sheep artificial insemination: A particular insight. Reprod Dom Anim, v.54 (suppl.4), p.32-40, 2019.

Anel L, Alvarez M, Martinez-Pastor F, Garcia-Macias V, Anel E, de Paz, P. Improvement strategies in ovine artificial insemination. Reprod Dom Anim, v.41 (suppl.2), p.30-42, 2006.

Barnes FL. The effects of the early uterine environment on the subsequent development of embryo and fetus. Theriogenology, v.53, p.649-658, 2000.

Campbell JW, Harvey TG, McDonald MF, Sparksman RI. Transcervical insemination in sheep: an anatomical and histological evaluation. Theriogenology, v.45, p.1535-1544, 1996.

Casali R, Pinczak A, Cuadro F, Guillen-Muñoz JM, Mezzalira A, Menchaca A. Semen deposition by cervical, transcervical and intrauterine route for fixed-time artificial insemination (FTAI) in the ewe. Theriogenology, v.103, p.30-35, 2017.

Croy BA, Prudencio J, Minhas K, Ashkar AA, Galligan C, Foster RA, Buckrell B, Coober, BL. A preliminary study on the usefulness of HUIL-8 in cervical relaxation of the ewe for artificial insemination and for embryo transfer. Theriogenology, v.52, p.271-287, 1999.

Dias FEF, Lopes Júnior ES, Villaroel ABS, Rondina D, Lima-Verde JB, Paula NRO, Feitas VJF. Sincronização do estro, indução da ovulação e fertilidade de ovelhas deslanadas após tratamento 
Alvares et al. Técnica de inseminação artificial por tração cervical e função luteal em ovelhas Santa Inês após estro sincronizado com cloprostenol ou progestágeno-eCG

hormonal com gonadotrofina coriônica equina. Arq Bras Med Vet Zootec, v.53, p.618-623, 2001.

Dobson, H, Fergani C, Routly JE, Smith RF. Effects of stress on reproduction in ewes. Anim Reprod Sci, v.130, p.135-140, 2012.

Emsem E, Gimenez-Dias C, Kutluca M, Koycegiz F. Reproductive response of ewes synchronized with different lengths of MGA treatments in intrauterine insemination program. Anim Repr Sci, v.126, p.57-60, 2011.

Falchi L, Scaramuzzi RJ. The expression of Era, OTR, cPLA 2, COX-2 and PPAR $\gamma$ in the cervix of the ewe during the estrous cycle. Theriogenology, v.79, p.40-47, 2013.

Fierro S, Olivera-Muzante J, Gil J, Viñoles C. Effects of prostaglandin administration on ovarian follicular dynamics, conception, prolificacy and fecundity in sheep. Theriogenology, v.76, p.630-639, 2011.

Halbert GW, Dobson H, Walton JS, Buckrell BC. The structure of the cervical canal of the ewe. Theriogenology, v.33, p.977-992, 1990

Hassani N, Ebrahimi M, Ghasemi-Panahi B, Hosseinkhani A. Evaluating reproductive performance of three estrus synchronization protocols in Ghezel ewes. Theriogenology, v.122, p.9-13, 2018.

Kershaw CM, Khalid M, McGowan MR, Ingram K, Leethongdee S, Wax G, Scaramuzzi RJ. The anatomy of sheep cervix and its influence on the transcervical passage of an inseminating pipette into the uterine lumen. Theriogenology, v.64, p.1225-1235, 2005.

Kershaw-Young CM, Scaramuzzi RJ, McGowan MR, Ptsillides AA, Wheeler-Jones CPD, Khalid M. The effect of estradiol on COX-2, EP2 and EP4 mRNA expression and the extracellular matrix in the cervix of the hypogonadotrophic, ovariectomized ewe. Theriogenology, v.73, p.620-628, 2010.

Manes J, Hozbor F, Alberio R, Ungerfeld R. Intravaginal placebo sponges affect negatively the conception rate in sheep. Small Rumin Res, v.120, p.108-111, 2014.

Martin GB, Ferasyi TR. Clean, Green, Ethical (CGE) management: what research do we really need? Int J Trop Vet Biomed Res, v.1, p.1-8, 2016.

Miranda VO, Oliveira FC, Dias JH, Vargas Júnior SF, Goularte KL, Sá Filho MF, Sá Filho OG, Baldassarre H, Vieira AD, Lúcia Jr T, Gasperin BG. Estrus resynchronization in ewes with unknown pregnancy status. Theriogenology, v.106, p.103-107, 2018.

Mitchell SE, Robinson JJ, King ME, McKelvey WAC, Williams LM. Interleukin 8 in the cervix of non-pregnant ewes. Reproduction, v.124, p.409-416, 2002.

Moura DS, Lourenço TT, Moscardini MM, Scott C, Fonseca PO, Souza FF. Aspectos morfológicos da cérvice de ovelhas. Pesq Vet Bras, v.31, p.33-38, 2011

Naqvi SMK, Joshi A, Bag S, Pareek SR, Mittal JP. Cervical penetration and transcervical AI of tropical sheep (Malpura) at natural oestrus using frozen-thawed semen. Small Rumin Res, v.29, p.329333, 1998.

Olivera-Muzante J, Fierro S, López V, Gil J. Comparison of prostaglandin- and progesterone-based protocols for timed artificial insemination in sheep. Theriogenology, v.75, p.1232-1238, 2011.

Purdy PH, Spiller SF, McGuire E, McGuire K, Koepk K, Lake S, Blackburn HD. Critical factors for non-surgical artificial insemination in sheep. Small Rumin Res, v.191, p.106179, 2020.

Richardson L, Hanrahan JP, Donovan A, Martí JI, Fair S, Evans ACO, Lonergan P. Effect of site of deposition on the fertility of sheep inseminated with frozen-thawed semen. Anim Reprod Sci, v.131, p.160-164, 2012.

Rosasco SL, Beard JK, Hallford DM, Summers AF. Evaluation of estrus synchronization protocols on ewe reproductive efficiency and profitability. Anim Reprod Sci, v.210, p.106191, 2019.

Schäfer-Somi S. Cytokines during early pregnancy of mammals: a review. Anim Reprod Sci, v.75, p.7394, 2003.

Weems CW, Weems YS, Randel RD. Prostaglandins and reproduction in female farm animals. Vet J, v.171, p.206-228, 2006.

Wiley TM, Cárdenas H, Pope WF. Effect of the rate of progesterone decline at luteolysis on the ovulatory follicles and subsequent estrous cycle length in ewes. Anim Reprod Sci, v.46, p.78-87, 1997.

Wulster-Radcliffe MC, Lewis GS. Development of a new transcervical artificial insemination method for sheep: effects of a new transcervical artificial insemination catheter and traversing the cervix on semen quality and fertility. Theriogenology, v.58, p.1361-1371, 2002.

Wulster-Radcliffe MC, Wang S, Lewis GS. Transcervical artificial insemination in sheep: effects of a new transcervical artificial insemination instrument and traversing the cervix on pregnancy and lambing rates. Theriogenology, v.62, p.990-1002, 2004. 